\title{
Residual and cyclic stresses in automotive plain bearings
}

\author{
S. Syngellakis ${ }^{1} \&$ A. Burke-Veliz ${ }^{2}$ \\ ${ }^{1}$ Wessex Institute of Technology, UK \\ ${ }^{2}$ Tecnologico de Monterrey, Campus Toluca, Mexico
}

\begin{abstract}
This paper presents a review of existing methodologies for predicting stress and strain development in automotive plain bearings. Initially, it deals with the modelling of the forming and fitting operations, leading to the determination of residual stresses, then with the numerical processes of generating cyclic stresses and plastic strains due to the reciprocating engine forces. Modelling tools as well as their adopted assumptions and simplifications are explained. Particular attention is given to the distinction between and effects of hydrodynamic and elastohydrodynamic lubrication conditions. The output of such analyses is illustrated through the inclusion of some typical results; its significance is discussed in the context of bearing design against fatigue failure.

Keywords: plain bearings, stress analyses, bearing forming, bearing fitting, elastohydro dynamic lubrication.
\end{abstract}

\section{Introduction}

The design of automotive plain bearings is a complex process involving the consideration of manufacture, assembly and service conditions. Adopted design strategies are based on the consideration of three major contributors to stress development: forming and fitting of the bearing in its housing, internal forces in the engine and hydrodynamic oil film pressure. The assessment of the latter is particularly challenging since it is coupled with the elastic deformation of the housing and the journal. Complex and rapidly changing loading patterns develop over the lining surface as the engine cycle goes through the different combustion stages. The resulting film pressure is characterized by steep gradients causing 
periodic stress concentrations superimposed on residual stresses caused by the manufacturing and assembly processes.

Reliable predictions of material response contribute to the understanding of the possible reasons for fatigue crack initiation and growth. Stress and strain estimates can be used as key indicators of the endurance of the component. The objective of this paper is to review recent progress in assessing the effects of bearing assembly and service conditions on the material. The emphasis in the review is on the methodologies for generating stress and deformation predictions rather than the results themselves, which obviously depend on specific bearing architectures and materials as well as boundary conditions. Plain bearings are cylindrical shells consisting of a flexible lining and stiff backing layers. The lining itself can be a multi-layered assembly of various metallic substances. In recent years, the automotive industry mostly uses various aluminium alloys as lining materials; the results presented here have been obtained using material and geometric data from such bearings.

The influence of manufacturing and assembly processes on bearing performance was not accounted for in early fatigue studies. The incorporation of the forming process and interference fit, in particular, into an integrated modelling procedure is therefore an area deserving particular attention. In early bearing analyses under service conditions, the geometry, material properties and loading were simplified; influences of plasticity, elastohydrodynamic lubrication (EHL) as well as the dimensionality of fluid pressure were recognised and subsequently addressed in more sophisticated modelling approaches.

\section{Residual stresses}

\subsection{Forming}

A concave bearing shell is formed through a punch driving a flat strip into a die. This is often followed by two secondary processes known as coining and broaching. Coining is the application of a membrane strain of approximately 0.005 through a tangential displacement of the shell ends when the punch is located at its lowest position in contact with most of the lining surface. This operation is aimed at reducing the intensity of tensile residual stresses. Broaching is the removal of approximately the top $25 \%$ of the lining layer so that geometrical tolerances are fulfilled. In the case of aluminium alloy lining, the removed material includes a thin, pure aluminium protective layer.

The forming process is clearly a mechanical problem with a high level of geometric and material non-linearity. Recently published descriptions of finite element (FE) simulations of this problem are summarised here $[1,2]$; no earlier record of such modelling work could be found in the published literature. The developed FE models accounted for three different types of non linearity: elastoplasticity, large deformation and contact between the forming tools and the strip. Both die and punch were modelled as rigid due to their stiffness being much higher than that of the flat strip. The punch was displaced towards the die forcing the strip into a semi-cylindrical shape; when the punch was removed, spring and 
frictional forces were still keeping the formed bearing attached to the die. The final residual stresses were obtained by forcing the formed bearing to follow the removed punch thus freeing it completely from the die.

The stresses predicted from a three-dimensional (3D) forming analysis were almost constant across the bearing width; this led to the adoption of twodimensional (2D) modelling allowing for a greater mesh density, especially in the backing. A dynamic friction coefficient $\mu=0.1$ between the surfaces in contact was initially adopted; this is comparable to that for two lubricated components of steel. The tensile residual tangential stress was found constant over most of the lining surface, especially in the lowest central part of the concave shell.

The influence of $\mu$ on the predictions was assessed by running the analysis with various values in the range from 0 to 0.25 . The results for the residual tangential stress shown in fig. 1 were obtained using the following data: radiuses of the punch and die set equal to 27.105 and $28.215 \mathrm{~mm}$, respectively; strip length of $95.61 \mathrm{~mm}$, strip width of $29.49 \mathrm{~mm}$, lining thickness of $0.42 \mathrm{~mm}$ and backing thickness of $1.8 \mathrm{~mm}$. Information on the elasto-plastic properties used can be found in the original article [2].

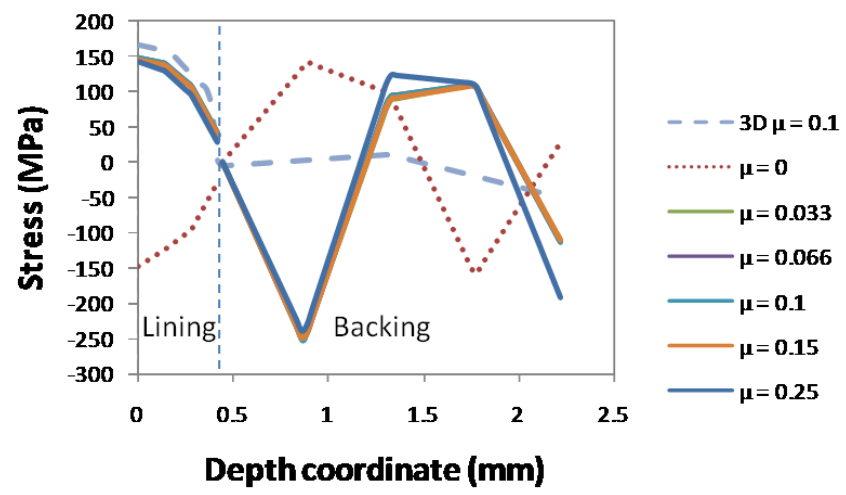

Figure 1: Dependence of tangential residual stress on friction.

It can be noted in fig. 1 that the residual stress results from $2 \mathrm{D}$ and $3 \mathrm{D}$ analyses for $\mu=0.1$ are very similar, especially in the lining where greater mesh density was used; they are significantly different from those obtained when friction is ignored. In the frictionless simulation, contact between punch and strip shifted to two outer zones, away from the centre, as the punch was driven downwards. This is in contrast with all analyses with friction, where contact remained mostly over the central region. The predicted residual stresses show little dependence on $\mu$ within the range from 0.03 to 0.25 .

\subsection{Fitting}

The bolting-up or assembly process comprises two actions: (i) spread, which is the forcing or snapping of the semi-elliptical bearing half-shell into the semi- 
circular housing and (ii) crush, which is the interference fit of the bearing into the housing due to the parting edges of each half shell protruding slightly above the bore parting edges. Bearing crush also results in an inward radial displacement of the lining surface in the region close to the split lines of the bearing. This reduces the clearance between the journal and the bearing in that region. Both actions cause compressive stresses in the lining of the bearing, which will reduce the tensile residual stresses due to cold forming thus affecting the elastic shakedown limit.

The assembly process has been simulated through a non-linear FE contact analysis [3] that estimates the bearing shape and clearances between the bearing and journal surfaces. This was part of an overall bearing-housing analysis that included the effects of elasto-hydrodynamic lubrication (EHL). No stress details specific to the bolting-up process were given. Such results have been presented [4] separately for spread and crush without however accounting for the forming residual stresses.

The state of stress at the end of the bolting-up process is essentially the same as that predicted at the end of coining during the forming process. A more detailed description of the results from a forming simulation that included coining and broaching, performed with $\mu=0.1$ [1], is presented in fig. 2. The "after coining" step result should describe very closely the state of stress in the bearing after bolting-up. It is noted in fig. 2 that the broaching simulation did not affect much the final residual stresses; it is therefore reasonable to assume that broaching would not have a significant effect on the bolting-up stresses. These results confirm the earlier assertion that bolting-up significantly reduces the residual tensile tangential stress. Circumferential variation of this stress showed that it remains tensile over certain zones close to the edge of each half-shell [2].

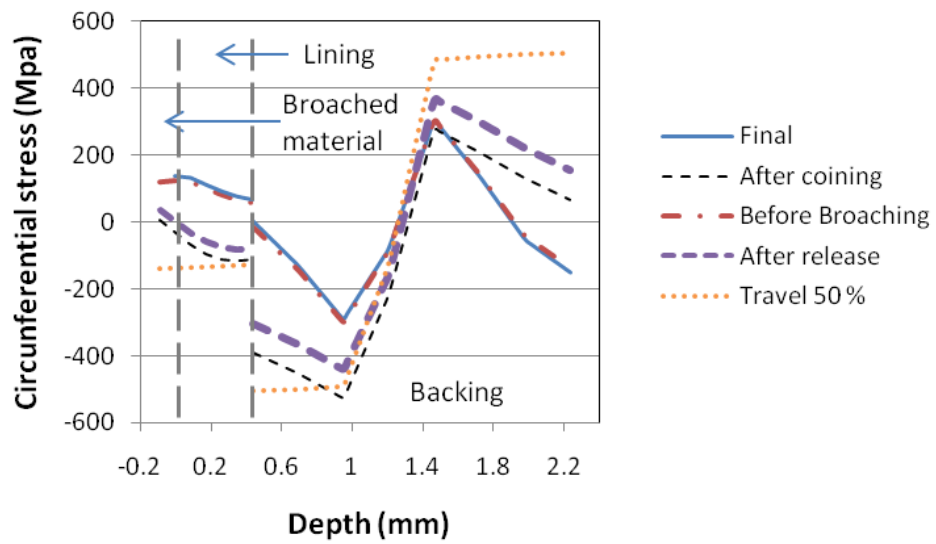

Figure 2: Tangential residual stress at various stages of the forming process. 


\section{Cyclic stresses}

\subsection{Hydrodynamic lubrication}

The reciprocating forces are transmitted to the bearing through the oil film between the journal and the bearing surface. Due to the non-uniform distribution of the oil film pressure as well as the complexity of assessing the effects of housing flexibility and crankshaft motion, it is very difficult to determine, with a reasonable degree of accuracy, cyclic bearing stresses that may be linked to fatigue failure.

The oil film pressure distribution over the surface of the bearing lining can be obtained by a hydrodynamic analysis assuming that the bearing and shaft are rigid [5]. Such analyses exist for a complete, unbroken as well as a ruptured lubricant film [6]. An unbroken film would cause negative pressure, which has been detected experimentally under moderate steady load and low eccentricity ratios [7]. It has been argued elsewhere [5] that the film can only support a very small negative pressure before rupture occurs. Simplified methods for the determination of hydrodynamic pressure [6] assume that the extent of positive pressure regions is the same for complete and ruptured films. A special algorithm is required to deal with the non-linearity caused by film rupture or cavitation.

The amplitude of the oil film pressure is considered an important bearing durability factor [8]. There have been various attempts to link pressure directly to fatigue but it has been recognised that the stresses developing in the bearing lining are ultimately responsible.

Experimental evidence of axial cracks away from the bearing edges led to the investigation of tangential stresses through the analysis of the middle section of the cylindrical bearing using plane strain elasticity theory [9]. The bearinghousing assembly was roughly approximated as a two-layered ring system, the applied pressure was represented by a Fourier series and the results for stresses and displacements were obtained in the same form. A further simplification was based on the assumption that only the deformation of the more rigid housing is significant. Once the latter is determined, it can then be imposed on to the more compliant bearing to yield the critical stress cycles.

The FE method has been found more versatile in dealing with complex geometric features and boundary conditions. Bearings have been modelled as flat strips in simplified two-dimensional and, to a lesser extent, three-dimensional FE analyses. Both backing and lining materials were assumed elastic. Martin et al. [10] adopted a two-dimensional model equivalent to a length of $140^{\circ}$ - bearing arc using the dimensions of the bearings fatigue-tested by Gyde [11]. The applied positive hydrodynamic pressures were calculated from loading conditions in Gyde's experiments using short bearing theory; the constraints were representative of rigid housing conditions. Hacifazlioglu and Karadeniz [12] analysed a semi-infinite strip initially under plane strain conditions; then they extended their analysis to three dimensions assuming a parabolic pressure distribution along the longitudinal axis of the bearing. An additional layer in frictionless contact with the backing represented an elastic housing support. 
Elastic bearing shell models have also been developed and analysed under plain strain conditions. Using geometric and loading input based on Blundell's test data [13], the oil film pressure distribution was determined and applied for a sufficient number of times during the loading cycle to provide the stress variations in the lining [14]. Perez et al. [15] performed parametric studies to assess the effects of housing rigidity as well as backing stiffness and lining thickness on tangential stresses.

\subsection{Elasto-hydrodynamic lubrication}

An EHL analysis accounts for the local deformation of bearing surface as well as the dynamic distortions of crankshaft and bearing housing. In both cases, the coupled lubrication and structural problems need to be solved. The oil film pressure and the housing deformation are the EHL analysis outputs which enter into a subsequent stress analysis of the bearing shell. The minimum film thickness is also relevant in order to investigate if contact and its resulting shear over the lining surface may be responsible of crack initiation.

The EHL problem has been solved in the case of plain bearings using FE modelling [5]. Based on this EHL solution, Bahai and $\mathrm{Xu}$ [3] predicted the bearing stresses through an elasto-plastic FE model comprising a simplified representation of the housing and the bearing shell. They used a special element formulation for axisymmetric geometry combined with Fourier interpolation relative to the circumferential direction. Hence, this was a $3 \mathrm{D}$ analysis but symmetric with respect to middle circumferential section. The stress output included the effects of interference fit.

More recently, the strategy of separating the stress analysis of the bearing from the global EHL modelling was adopted [2]. The input to the bearing stress analysis was provided by an industry-developed and run EHL software [16], which solves the Reynolds equation by the finite difference method and accounts for the elastic deformation of both journal and bearing-rod assembly. This EHL analysis assumes the absence of bubble collapse within the fluid and may also involve thermal responses, that is, include a heat balance analysis to determine the oil viscosity and material expansion in order to estimate with greater accuracy the clearances between bearing and journal. The analysis may be extended to include the modelling of oil feed, journal profile (including grooves and elliptical shapes) and solid-to-solid contact with flexible components.

In order to account for the residual forming stresses, the mesh used in the EHL analysis was consistent to that of the flat strip subjected to the forming and fitting process described in section 2; this facilitated the transfer of residual stress information to the model subjected to the EHL pressure and the deformation of the bearing casing. The latter was obtained by multiplying the EHL nodal pressures by the compliance matrix of the housing and bearing. It is then possible to estimate the vector of nodal radial displacements $\mathbf{d}_{c}$ at the interface between backing and casing from

$$
\mathbf{d}_{c}=\mathbf{d}_{b}+\mathbf{d}_{f}+\mathbf{d}_{n}
$$


where $\mathbf{d}_{b}, \mathbf{d}_{f}$ and $\mathbf{d}_{n}$ represent, respectively, the nodal radial displacement vectors caused by the reciprocating engine forces, the fitting operations and the EHL pressure [3]. Fig. 2 illustrates the application of kinematic and pressure boundary conditions to the solid model of the bearing.

\subsubsection{Accelerated fatigue test}

Bearing stresses were obtained under EHL loading conditions corresponding to accelerated fatigue tests on a rig consisting of an eccentric shaft that forces a piston to travel in the vertical direction through a connecting rod [17]. At the same time, the rig applies some resistance to the movement of the piston due to the oil present in the chamber of a hydraulic ram. This oil is forced into another chamber and its flow is regulated by valves to obtain the desired resistance of travel or load applied to the bearing. The bearing casing used in these tests is stiffer than the regular casings used in automotive engines leading to conditions that promote extended lives in tested bearing.

Due to the nature of the rig, the specific load (defined as the piston load divided by the projected bearing area) is applied on the bottom shell; however, casing deformations deform the upper bearing shell as well. The evolution of deformation and pressure during the engine cycle is shown in figs. 3(a) and 3(b), respectively, on the bearing symmetry plane. The EHL pressures and bearing deformations were obtained for tests at $3000 \mathrm{rpm}$ and a specific load of $97 \mathrm{MPa}$. The bearing dimensions are those given in section 2.1 .

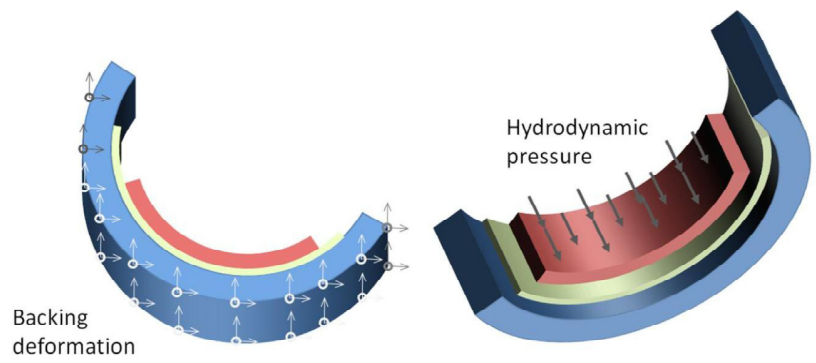

Figure 3: Schematic illustration of the bearing model and applied loading.

Both elastic and elasto-plastic analyses were performed; results were obtained with and without the residual stresses being accounted for. Neglecting the residual stresses was justified by the possible stress relief caused by the service temperature (around $150^{\circ} \mathrm{C}$ ). It is also possible that the work hardening process occurring in the first few cycles may cause an elastic shakedown followed by elastic deformations in subsequent cycles.

Since the results from elastic-plastic analyses depend on the previous load history, the cyclic strain amplitudes were estimated over three loading cycles; this proved sufficient to reaching a stable deformation cycle.

Greater strains and stresses were predicted in the central part of the bottom bearing shell near the EHL pressure peak, which occurs at a crank angle of approximately $180^{\circ}$. Stress predictions were considerably affected by the 
inclusion of residual stresses while strains were affected to a lesser extent. The tangential stress variations with time (crank angle) and angular location, at an axial section $1.8 \mathrm{~mm}$ from the bearing edge, obtained from the elasto-plastic analyses, are shown in fig. 4.

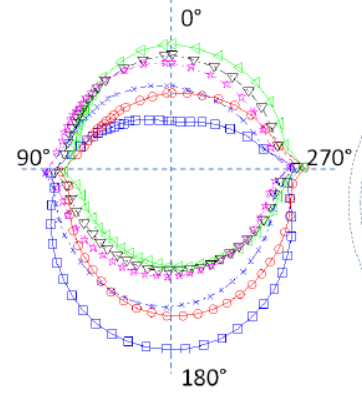

(a)

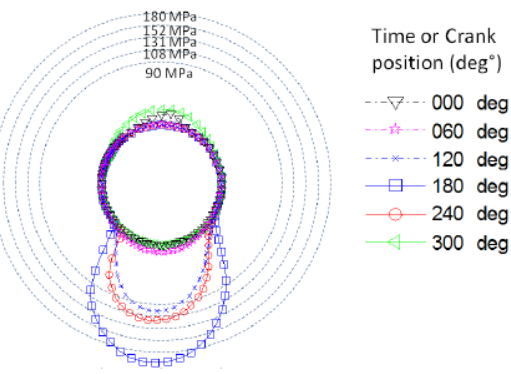

(b)

Figure 4: (a) Radial displacement at backing-casing interface, (b) oil film pressure during an accelerated fatigue test cycle.

The elasto-plastic model predicted that the lining surface remained under compressive stresses during most of the engine cycle; these stresses were more pronounced when residual stresses were not accounted for. Despite the existence of mainly compressive stresses, positive circumferential strains of slightly greater magnitude than the ones observed in the linear elastic model were calculated.

\subsubsection{Engine conditions}

FE modelling was also carried out for a bearing subjected to the loading and constraint input arising from a real engine (Puma) operating at $1800 \mathrm{rpm}$ [1]. The bearing geometry and material properties were different from those considered for the accelerated fatigue test. The same industrial software [16] provided the oil film pressure and housing deformation arising under EHL conditions. In agreement with experimental evidence, the top shell was found to be subjected to higher specific load and therefore more prone to damage. Results were obtained assuming elastic behaviour and ignoring forming and fitting residual stresses.

The highest pressure in the axial direction developed at a small distance from one of the bearing edges and results were plotted at the corresponding axial section focusing on tangential and shear strain. It was interesting to note positive tangential strain cycles developing over most of the top bearing shell along its circumferential direction. Their maximum was observed at a crankshaft angle of approximately $400^{\circ}$ and a bearing angular position of approximately $180^{\circ}$ (with $0^{\circ}$ corresponding to the middle of the bottom shell). The significant levels of predicted shear strain pointed to crack growth under mixed mode conditions; this is consistent with the oblique crack paths observed in failed bearings subjected to engine test conditions. 


\subsection{Sub-modelling}

A fatigue crack growth investigation based on damage tolerance principles would require a more detailed description of the stress and strain states within the lining layer; this can be obtained through an FE technique known as submodelling. The sub-modelling process consists of several steps involving a full and sectioned model. First, a solid model and mesh of part of the component should be created ensuring that the node numbering at the interface, where connecting material is located in the full model, would remain fixed. Then, nodes placed at the border are selected and exported along with their coordinates. The node number and coordinates are imported into the solution of the full analysis. Full and sub-model should share a common origin and coordinate system. The process is schematically illustrated in fig. 5. A severe limitation of a submodelling analysis is its relevance to only elastic material models.

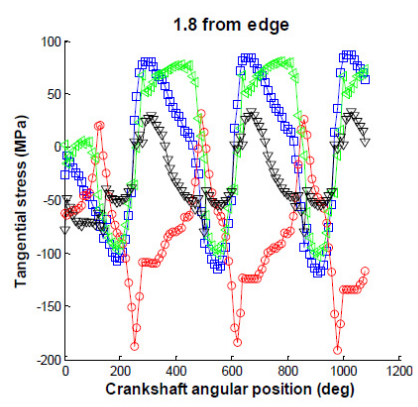

(a)

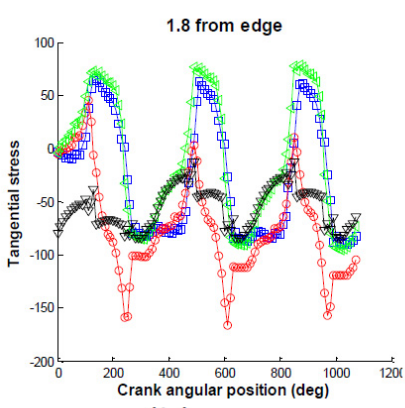

(b)

Figure 5: Tangential stress vs. crank angle predicted by the elastic-plastic analysis; (a) neglecting and (b) accounting for residual stresses.

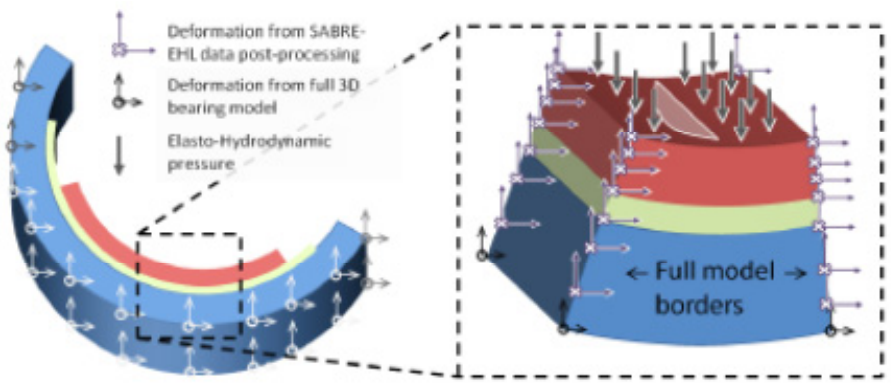

Figure 6: Schematic illustration of sub-modelling for bearing stress analysis.

Sub-modelling was attempted in the context of the recent bearing analysis described in the previous sections [1]. The EHL pressure was applied to the submodel by dividing the bearing surface into a grid and specifying an average 
pressure value to the nodes found within the grid. This process, along with the transfer of the interface conditions, was carried out consecutively for every solution step. It is important to note that sub-modelling predictions near the interfaces, where boundary conditions from the full model were transferred, are not usually accurate. The solution domain of interest should be placed away from these interfaces.

\section{Conclusions}

Some early conclusions were drawn from the stress analyses of bearings assuming simply hydrodynamic lubrication, that is, no interaction between oil film pressure and housing deformation. Negative oil film pressure may arise but its effect on predicted stresses was not systematically assessed; it was argued that the consistency between theoretical predictions and experimental measurements may be improved if negative pressure is taken into account [7].

Tensile tangential stresses were found where the pressure falls to zero, that is, at the location of steepest pressure gradient; their maximum value was predicted at the backing-lining interface [10]. Both flat strip [10] and a bearing shell [14] analyses showed good correlation between the fatigue crack origins in test specimens $[11,13]$ and their assessments of the maximum tangential stress. However, shear stress and distortion energy maxima were found subsurface. Parametric studies indicated that housing rigidity would have the greatest influence on the magnitude of the tensile stresses that develop at the surface of the lining. These stresses as well as the shear stresses at the lining-backing interface rise with decreasing housing stiffness and increasing lining thickness [15].

The coupled analyses under EHL conditions performed by Bahai and Xu [3] lead to the conclusion that tensile deviatoric tangential stresses are present at the observed crack initiation site during the whole engine cycle while their hydrostatic counterpart dominates the overall stress state in a compressive environment. A more recent, multi-step plain bearing modelling procedure [1, 2], accounting for all causes of stress development, produced stress and strain cycles with the potential to initiate fatigue damage. Forming and fitting residual stresses were found to have a significant impact on the stress results although strains were affected to a lesser extent. The high plastic deformation predicted by the numerical analyses corresponded to high cycle fatigue; this however was not consistent with fatigue test results and led to the consideration of engine temperature and elastic shakedown as possible reasons for milder stress conditions and the use of simpler elastic analyses. The damage maps based on predicted strain and stress amplitudes were consistent with experimental observations.

The effectiveness of sub-modelling technique was tested under accelerated test conditions; the results obtained were in good agreement with those from the full model. The generation of such sub-models can be very useful in damage tolerant analyses since the creation of a full model incorporating a propagating crack would require enormous memory resources and computation times [18]. 


\section{Discussion}

Bearing design requires the analysis of multi-layer architectures; this can only be achieved by FE modelling of the respective elastic or elasto-plastic problem, especially when EHL would be taken into account. Using the predictions of housing deformation and oil film pressure obtained under EHL conditions, it is possible to predict the elasto-plastic behaviour of the materials used in the different layers; this can lead to a reliable assessment of the fatigue performance of bearings.

Validation of FE analyses is essential to their reliability. With regard to forming and fitting stresses, analytical solutions may be obtained under simplified conditions for that purpose. Experimental measurement of residual stresses could also provide confidence in the analytical results as well as information on possible stress relief due to in-service heating. Stress analyses that account for cold forming have indicated that the resulting residual stresses may play a role in bearing failure.

The complexity of the overall bearing stress analysis raises the need for the examination of possible limitations in the general purpose FE software used in previous modelling attempts. A long term strategy would be the development of a dedicated code combining FE modelling for the global EHL problem with boundary element (BE) modelling for the local bearing stress problem. The modelling could be further extended to include discontinuous oil islands, which may set up tensile stresses on the surface of the lining.

Previous damage tolerance approaches were based on simple states of stress arising in flat strips under three-point bending. In contrast, the actual state of stress observed in bearings under service conditions is three-dimensional and this may reduce the validity of crack growth results obtained under bending conditions [18]. The complexity of the state of stress may be enhanced by other factors such as service temperature, penetration of oil into the crack and solid to solid contact.

It can be argued that damage tolerance approaches would not be suitable to bearing design in an industrial environment. The developed analyses may provide extensive information on the cyclic deformation experienced by the bearing material but, as mentioned above, they still do not account, either directly or indirectly, for other important damage mechanisms arising during the bearing life.

\section{References}

[1] Burke-Veliz, A., Finite element modelling of fatigue crack growth in multilayered architectures Ph.D. Thesis, University of Southampton, 2009.

[2] Burke-Veliz, A., Wang, D., Wahdy, N., Reed, P.A.S., Merritt, D. and Syngellakis, S., Plain bearing stresses due to forming and oil film pressure. Journal of Physics: Conference Series, 181, 012010, 2009.

[3] Bahai, H. and $\mathrm{Xu}, \mathrm{H}$., Three-dimensional elastoplastic finite element and elastohydrodynamic analyses of journal bearings. Proceedings of the 
Institution of Mechanical Engineers Part C: Journal of Mechanical Engineering Science, 211, pp. 143-152, 1997.

[4] Thomazi, C., Pérez, M., Villar, M.M. and Syngellakis, S., Analysis of precision insert bearing interference fit. $14^{\circ}$ POSMEC - Simpósio do Programa de Pós-Graduação em Engenharia Mecânica, Universidade Federal de Uberlândia, 2004.

[5] McIvor, J.D.C. and Fenner, D.N., Finite-element analysis of dynamically loaded flexible journal bearings - a fast Newton-Raphson method. Journal of Tribology-Transactions of the ASME, 111(4), pp. 597-604, 1989.

[6] Booker, J.F., Dynamically loaded journal bearings: mobility method of solution. Trans. ASME, J. Basic Eng., 187, pp. 537-546, 1965.

[7] Dyer, D. and Reason, B.R., A study of tensile stresses in a journal-bearing oil film. J. Mech. Eng. Sci., IMechE, 18, pp. 46-52, 1976.

[8] Ushijima, K., Aoyama, S., Kitahara, K., Okamoto, Y., G, J. and Xu, H., A study on engine bearing wear and fatigue using EHL analysis and experimental analysis. Advances in Powertrain Tribology (SP-1463), Society of Automotive Engineers: Warrendale, PA, pp. 1999.

[9] Lang, O.R., Surface fatigue of plain bearings. Wear, 43(1), pp. 25-30, 1977.

[10] Martin, F.A., Garner, D.R. and Adams, D.R., Hydrodynamic aspects of fatigue in plain journal bearings Journal of Lubrication TechnologyTransactions of the ASME, 103(1), pp. 150-156, 1981.

[11] Gyde, N., Fatigue fractures in babbitt lined journal bearings, Ph.D. Thesis, Technical University of Denmark, 1969.

[12] Hacifazlioglu, S. and Karadeniz, S., A parametric study of stress sources in journal bearings. International Journal of Mechanical Sciences, 38(8-9), pp. 1001-1015, 1996.

[13] Blundell, J.K., Fatigue initiation in thin-walled journal bearings. ASLE Transactions, 23(2), pp. 131-140, 1980.

[14] McCallion, H. and Lotfi, M., Tensile surface stresses and fatigue in plain journal bearings. Tribology International, 25(4), pp. 247-257, 1992.

[15] Perez, M., Thomazi, C. and Syngellakis, S., Stress analysis of fluid-film bearings. Proceedings of the International Conference on Gearing, Transmissions, and Mechanical Systems, ed. D. Su, Professional Engineering Publishing: pp. 729-738, 2000.

[16] Mian, A.O., Merritt, D. and Wang, D., The effect of crankshaft flexibility on the EHL of connecting rod bearings. SAE 2002 World Congress, Detroit, Michigan, 2002.

[17] Xu, H., Jones, G.J., Aoyama, S., Ushijima, K., Okamoto, Y. and Kitahara, K., Simulation of bearing wear and its influence upon bearing performance based on elastohydrodynamic analysis. SAE 1999 World Congress, Detroit, Michigan, 1999.

[18] Burke-Veliz, A., Syngellakis, S. and Reed, P.A.S., Fatigue crack shielding and deflection in plain bearings under large-scale yielding. Engineering Failure Analysis, 17(3), pp. 648-657, 2010. 